\title{
Experimental evidence of the rear capture of aerosol particles by raindrops
}

\author{
Pascal Lemaitre $^{1}$, Arnaud Querel ${ }^{2, a}$, Marie Monier ${ }^{3,4}$, Thibault Menard ${ }^{5}$, Emmanuel Porcheron $^{1}$, and \\ Andrea I. Flossmann 3,4 \\ ${ }^{1}$ Institut de Radioprotection et de Sûreté Nucléaire (IRSN), PSN-RES, SCA, LPMA, Gif-sur-Yvette, France \\ ${ }^{2}$ Institut de Radioprotection et de Sûreté Nucléaire (IRSN), PRP-CRI, SESUC, BMCA, Fontenay-aux-Roses, France \\ ${ }^{3}$ Clermont Université, Université Blaise Pascal, Laboratoire de Météorologie Physique, Clermont-Ferrand, France \\ ${ }^{4}$ CNRS, INSU, UMR6016, LaMP, Aubière, France \\ ${ }^{5}$ CNRS UMR6614, CORIA Rouen, Site Universitaire du Madrillet, Saint-Étienne-du-Rouvray, France \\ ${ }^{a}$ currently at: Strathom Energie, Paris, France \\ Correspondence to: Pascal Lemaitre (pascal.lemaitre@irsn.fr)
}

Received: 1 December 2016 - Discussion started: 5 January 2017

Revised: 4 March 2017 - Accepted: 7 March 2017 - Published: 28 March 2017

\begin{abstract}
This article presents new measurements of the efficiency with which aerosol particles of accumulation mode size are collected by a $1.25 \mathrm{~mm}$ sized raindrop. These laboratory measurements provide the link to reconcile the scavenging coefficients obtained from theoretical approaches with those from experimental studies. We provide here experimental proof of the rear capture mechanism in the flow around drops, which has a fundamental effect on submicroscopic particles. These experiments thus confirm the efficiencies theoretically simulated by Beard (1974). Finally, we propose a semi-analytical expression to take into account this essential mechanism to calculate the collection efficiency for drops within the rain size range.
\end{abstract}

\section{Introduction}

Aerosol particles are important components of the atmosphere. They contribute significantly to the Earth's energy budget by interacting with solar radiation directly as well as indirectly by serving as precursors to cloud formation (cloud condensation nuclei- $\mathrm{CCN}$ ) which also will interact with this radiation (Twomey, 1974). Furthermore, the physical properties of these particles in suspension within the atmosphere (size, concentration, affinity for water, etc.) are essential parameters for characterising air quality. For these reasons, the scientific community has actively studied the physics of atmospheric aerosol particles.

There are several aerosol particles origins. The primary natural sources are sea spray, wind-driven dust, volcanic eruptions and human activities. The secondary sources are associated with the gas-to-particle conversion of certain gases present in the atmosphere. The size of these particles greatly varies and ranges from $1 \mathrm{~nm}$ to several hundred microns. Particles of anthropogenic origin represent an increasingly large proportion of aerosol particles in the atmosphere (Charlson et al., 1992; Wang et al., 2014). Among all anthropogenic pollutants, radioactive releases from a nuclear accident are of high risk for both humans and the environment. Just like all other particles, once emitted, radioactive particles undergo physical processes that drastically change their size distribution during their transport in the atmosphere. Ultrafine particles are very sensitive to Brownian diffusion and grow by coagulation. Large particles settle on the ground due to gravity. Hence, there is a particle size range that has no efficient removal process and a very long atmospheric residence time. This size range is referred to as the accumulation mode (Whitby, 1973) and comprises particles with a diameter between 0.1 and $2 \mu \mathrm{m}$. These particles can remain in the upper troposphere for several months (Jaenicke, 1988) and can be transported over long distances, crossing oceans and continents (Pruppacher et al., 1998). 
The accumulation of particles within this size range is essentially limited by two atmospheric processes: in-cloud scavenging (rainout) and below-cloud scavenging (washout) during rainfall events. Thus, in the event of a nuclear accident with a release of radioactive aerosol particles, it is essential to correctly model both of these mechanisms in order to predict their number concentration within the troposphere as well as the ground contamination.

This study focuses on the below-cloud scavenging of aerosol particles by rain with a microphysical approach. We aim to measure, in a laboratory setting, the collection efficiency of the aerosol particles constituting the accumulation mode, by drops of a size representative of rain. Recent measurements with $2 \mathrm{~mm}$ drops (Quérel et al., 2014b) have shown that, for submicron particles, the collection efficiency increases very rapidly when the size of the particles is reduced. The Slinn (1977) model does not reproduce this increase in efficiency, leading to errors of several orders of magnitude for the collection efficiency. We impute this discrepancy to the key hypothesis of the Slinn model which assumes Stokes flow conditions around the drop. Yet, since the Reynolds number of a $2 \mathrm{~mm}$ drop at its terminal velocity is approximately 800, this assumption of Stokes flow is unjustified. This model nonetheless remains the most common in the literature mainly because it is easy to use.

Quérel et al. (2014b) showed that the Beard (1974) model was the only one to predict this increase in the collection efficiency for submicron aerosol particles. However, direct measurements in the drop size range simulated by Beard (1974) could not be performed; the only comparison results from a linear extrapolation of theoretical computations to the measured size range. These efficiencies compared reasonably well even for aerosol particles in the submicron range. But the linear extrapolation is not completely satisfactory for an experimental validation of this model. This article provides experimental evidence of the robustness of Beard's simulation for the raindrop sizes under investigation in his paper, i.e. for diameters between 0.28 and $1.25 \mathrm{~mm}$.

Our paper is divided into three sections. First, we present a theoretical description of aerosol scavenging by rain. We then present our experimental setup and the associated experimental results. Finally, we compare our measurement results with the outcomes of the models of Beard (1974) and Slinn (1977) in order to propose a semi-empirical correlation for calculating the elementary collection efficiency associated with rear capture.

\section{Theoretical description of washout}

At mesoscale, the scavenging of aerosol particles by rain is described by the scavenging coefficient $(\lambda)$. This parameter is defined as the fraction of particles of diameter $d_{\text {ap }}$ captured by the raindrops per unit of time (Eq. 1). In this equation, $C\left(d_{\mathrm{ap}}\right)$ is the concentration of aerosol particles of diameter $d_{\text {ap }}$ in suspension in air per unit of volume.

$\frac{\mathrm{d} C\left(d_{\mathrm{ap}}\right)}{C\left(d_{\mathrm{ap}}\right)}=-\lambda_{\text {rain }}\left(d_{\mathrm{ap}}\right) \mathrm{d} t$

This parameter is essential for predicting air quality (Chate, 2005) and ground contamination following a nuclear accident with release of radionuclides into the environment (Groëll et al., 2014; Quérel et al., 2015). There are several approaches for determining this parameter. It can either be determined theoretically by solving Eq. (2) (Flossmann, 1986; Mircea and Stefan, 1998; Mircea et al., 2000) or measured in the environment by monitoring the variation of particulate concentration in the atmosphere during precipitation (Volken and Schumann, 1993; Laakso et al., 2003; Chate, 2005; Depuydt, 2013). $\lambda_{\text {rain }}\left(d_{\text {ap }}, D_{\text {drop }}\right)$ is defined by

$$
\begin{array}{r}
\lambda_{\text {rain }}\left(d_{\text {ap }}, D_{\text {drop }}\right)=\int_{D_{\text {drop }}=0}^{\infty} \frac{\pi D_{\text {drop }}^{2}}{4} \cdot U_{\infty}\left(D_{\text {drop }}\right) \\
E\left(d_{\text {ap }} D_{\text {drop }}, \mathrm{RH}\right) N\left(D_{\text {drop }}\right) \mathrm{d} D_{\text {drop }},
\end{array}
$$

where $D_{\text {drop }}$ is the drop diameter, $U_{\infty}\left(D_{\text {drop }}\right)$ is the terminal fall velocity, $N\left(D_{\text {drop }}\right) \mathrm{d} D_{\text {drop }}$ is the number concentration of drops with a diameter between $D_{\text {drop }}$ and $D_{\text {drop }}+\mathrm{d} D_{\text {drop }}$ during the rainfall event, and $E\left(d_{\mathrm{ap}}, D_{\text {drop }}, \mathrm{RH}\right)$ is the collection efficiency for a given drop size, particle size $\left(d_{\mathrm{ap}}\right)$ and relative humidity $(\mathrm{RH})$.

Unfortunately, these two approaches yield $\lambda_{\text {rain }}$ values that differ by several orders of magnitude, in particular for submicron particles (Laakso et al., 2003). It is clear, when we examine Eqs. (1) and (2), that each of the two methods has advantages and significant limitations, which are also highlighted by the authors. The main limitation for measurement of the scavenging coefficient in the environment remains the assumption that the change in concentration is exclusively related to collection by the drops. Even if the rainfall events are methodically selected, it is difficult to completely neglect advection, turbulent transport, coagulation and the influence of the hygroscopic behaviour of particle (Flossmann, 1991). For example, Quérel et al. (2014a) have recently shown that, during convective episodes, downdraft was the main cause of the reduction in particulate concentration, well before collection by the drops.

For the theoretical approach, the main limitation is the requirement to know the collection efficiency (Eq. 3). This microphysical parameter is defined as the ratio between the effective collection area (in other words, the cross-sectional area inside which the particle trajectory is intercepted by the drop) and the cross-sectional area of the drop. It is equivalent to defining the ratio of the mass of particles (of a given diameter) collected by the drop over the mass of particles (of the same diameter) within the volume swept by a sphere of equivalent volume (Eq. 3).

$E\left(d_{\mathrm{ap}}, D_{\text {drop }}, \mathrm{RH}\right)=\frac{m_{\mathrm{AP}, \text { collected }}\left(d_{\mathrm{AP}}\right)}{m_{\mathrm{AP}, \text { swept }}\left(d_{\mathrm{AP}}\right)}$ 
To compute this efficiency, one has to describe and model all the processes involved in the collection of particles by falling raindrops. Several mechanisms are usually considered, which are summarised hereafter; however, a more exhaustive review can be found in the literature (Pruppacher et al., 1998; Chate, 2005; Ladino et al., 2013; Ardon-Dryer et al., 2015). The three main mechanisms leading to this collection are Brownian motion, inertial impaction and interception. Small particles, with a radius on the order of the mean free path of the air molecules or smaller, are very sensitive to the collision of air molecules. Therefore, they shall deviate from streamlines due to Brownian motion. For large particles, with a diameter greater than $1 \mu \mathrm{m}$, their inertia prevents them from following the streamlines of the flow and they impact the drop on its leading edge. Aerosol particles with a diameter smaller than $1 \mu \mathrm{m}$ and much larger than the mean free path of the air molecules follow the streamlines of the flow around the drop. They might nevertheless enter in contact with the drop when the streamlines approach the drop at a distance smaller than the radius of the aerosol particle. For particles with diameter between 0.2 and $1 \mu \mathrm{m}$, there is a minimum collection efficiency called the "Greenfield gap" (Greenfield, 1957). For these particles, none of the three described mechanisms are efficient for collection. It is expected that phoretic forces would be the most efficient mechanisms. To be thorough, secondary mechanisms for collision are also described here. Thermophoresis and diffusiophoresis are, respectively, linked to thermal and water vapour gradients. The side of a particle exposed to warmer air is impacted by molecules with higher kinetic energy than molecules impacting the colder side. As a result, thermophoresis results in a force whose direction is the opposite of the thermal gradient. Similarly, particles exposed to a water vapour gradient are exposed to molecular collisions with a dissymmetric kinetic energy since water vapour molecules are lighter than air molecules. In the atmosphere, diffusiophoresis results in a force whose direction is the opposite of the water vapour gradient. Electro-scavenging could also have an important contribution when both droplet and aerosol particles are electrically charged, resulting in an attractive (or repulsive) force when they have opposite (or identical) polarity. Moreover, Tinsley et al. $(2000,2006)$ theoretically showed that electrically charged aerosol particles can induce an image charge on droplets that results in a short-range electrical attraction that increases collection efficiency even with neutrally charged droplets.

For each of these elementary mechanisms, theoretical expressions of the elementary collection efficiencies have been derived (Table 1).

Finally, the droplet total collection efficiency can be theoretically deduced by adding all these elementary collection efficiencies together. The use of these theoretical models seems justified for cloud droplets since they have very small Reynolds numbers. However, for raindrops with larger sizes and Reynolds numbers, there are many additional uncertain- ties. This is because, once they reach their terminal velocity, the Reynolds and Weber numbers of these large drops are very high. They thus oscillate at high frequency (Szakáll et al., 2010), which greatly complicates the simulation of flows inside and outside the drop. Furthermore, the boundary layer separation in the wake of the drop results in significant recirculating flows. Therefore, there are currently few methods for numerically simulating such flows (although the work of Menard et al., 2007, shall be mentioned). The most common approach continues to be to use the Slinn model (Volken and Schumann, 1993; Laakso et al., 2003; Chate, 2005; Depuydt, 2013), essentially for its ease of use and despite its simplifying assumptions. It should be kept in mind that Slinn models the flow around the drop as a Stokes flow, which results in ignoring the convective terms of the Navier-Stokes equation. Such flows have a similar kinematic field to that of a potential flow. The Slinn model cannot therefore capture the separation of the boundary layer in the wake of the drop. The flow on the front side of the drop is, however, relatively well modelled.

Beard and Grover (1974) have developed a numerical model that is more sophisticated than that of Slinn (1977) to numerically simulate the collision between particles and a drop. The main difference is that they do not assume Stokes flow. Flow around the drop is computed by solving the full Navier-Stokes equation including the convective term. However, Beard and Grover (1974) made two simplifying assumptions: the drop is assumed spherical and the flow axisymmetric. These simulations capture the separation of the boundary layer in the wake of the drop and the resulting recirculating flows. Using these simulations, Beard (1974) derived the collision efficiencies between drops and particles of different sizes. For this, he computed the particle trajectory in the flow considering drag and gravity forces. For the drag force, they followed the Stokes-Cunningham expression that takes into account non-continuum effects, which are important for the smallest particles. These simulations highlight, for the first time, the capture of submicron-sized particles in the rear of the drop, due to wake recirculations.

Until recently, no measurements in the numerous experimental studies (Kerker and Hampl, 1974; Grover et al., 1977; Wang and Pruppacher, 1977a; Lai et al., 1978; Pranesha and Kamra, 1996; Vohl et al., 1999) could be used to validate these two models since very few use submicron particles. Quérel et al. (2014a) showed that, for their dataset, the Slinn model underestimates by 2 orders of magnitude the measured collection efficiencies for particles with submicron sizes. As stated in the introduction, if they concluded that their data could confirm the Beard model, they were required to extrapolate the simulations of Beard to confront their observations.

In this paper, the collection efficiency is investigated experimentally for drops within the size range simulated by Beard (1974) to address these uncertainties in the collection efficiency of raindrops with large Reynolds numbers by ac- 
Table 1. References of theoretical expressions for the calculation of each collection mechanism.

\begin{tabular}{ll}
\hline Elementary mechanism & Reference \\
\hline Inertial impaction & Slinn (1977); Park et al. (2005) \\
Interception & Slinn (1977); Park et al. (2005) \\
Brownian motion & Slinn (1977); Park et al. (2005) \\
Diffusiophoresis & Waldmann (1959); Davenport and Peters (1978); \\
& Andronache et al. (2006); Wang et al. (2010) \\
Thermophoresis & $\begin{array}{l}\text { Davenport and Peters (1978); Andronache et al. (2006); } \\
\text { Wang et al. (2010) }\end{array}$ \\
Electro-scavenging & Davenport and Peters (1978); Andronache et al. (2006); \\
& Wang et al. (2010) \\
Image forces & Tinsley and Zhou (2015) \\
\hline
\end{tabular}

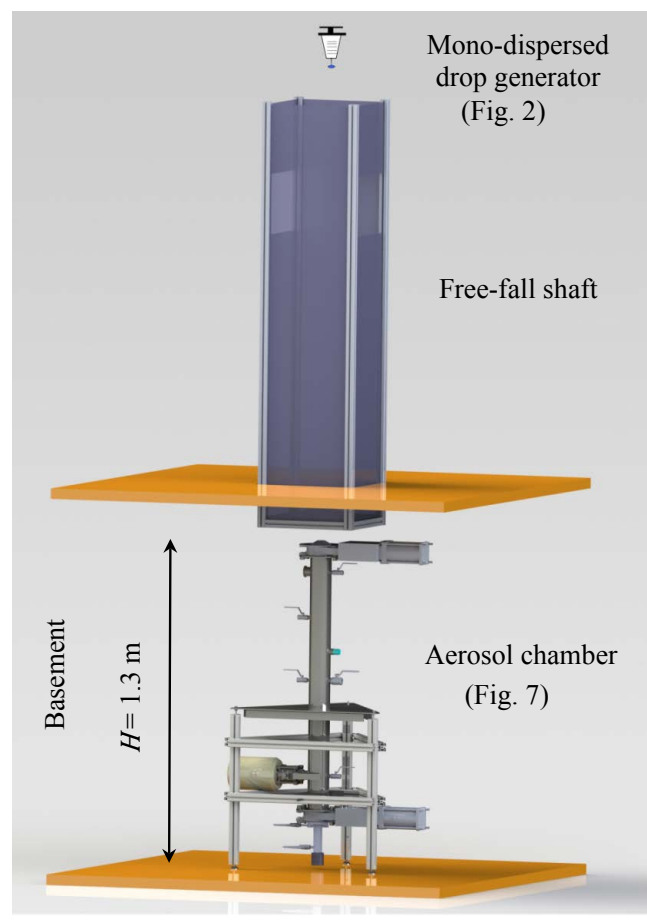

Figure 1. The new BERGAME facility.

curately measuring them in the laboratory with the ultimate aim of theoretically deriving a scavenging coefficient.

\section{Experimental facility}

The new experimental facility follows the one described and deployed by Quérel et al. (2014b). The equipment is called BERGAME (French abbreviation for a facility to study the aerosol scavenging and measure collection efficiency).

Presented in detail in the following subsections, the three stages are (Fig. 1)

- a mono-dispersed drop generator,

- a free-fall shaft and
- an aerosol chamber.

The main changes with respect to Quérel et al. (2014b) concern the drop generator and the aerosol chamber. Indeed, those authors concluded that drop generation has to be improved if direct comparisons with the Beard (1974) results were to be made. Improvements are presented in Sect. 2.1. In addition, the aerosol chamber has been modified not only to increase the particle number concentration but also to better control relative humidity, to neutralise the aerosol particles and to minimise uncertainties. The objective of these modifications is also to be consistent with the hypothesis of the Beard (1974) model, which considers only drag and gravitational forces on the aerosol particles. The modifications are thus intended to minimise electro-scavenging (discussed in Sect. 2.1 and 2.3), diffusiophoresis (discussed in Sect. 2.3 and Appendix A1) and thermophoresis. Both the drop generator and aerosol chamber are described in the following sections.

\subsection{Production of drops representative of rain}

In order to enable the generation of finer drops, a new generator (Fig. 2) was developed, characterised and installed at the top of the free-fall shaft of the BERGAME facility. The generator was placed $8 \mathrm{~m}$ above the new aerosol chamber. The total height of the drop shaft has been reduced by $2 \mathrm{~m}$ because, as the drops are smaller than those investigated by Quérel et al. (2014a and b), they reach their terminal velocity in a shorter distance.

The drop generator consists of a valve operated by piezoelectric actuators which transmit their movement to a rod. A ceramic sealing ball is attached to the rod and lifts to open the valve by enabling the fluid to flow (see Fig. 2). The water circuit is maintained under pressure by a compressed air system.

Classical piezoelectric drop-on-demand systems may produce electrically charged droplets (Ardon-Dryer et al., 2015). However, we want to limit electro-scavenging as Beard (1974) did in his simulations. To control electroscavenging, the net charge of each drop produced by this 

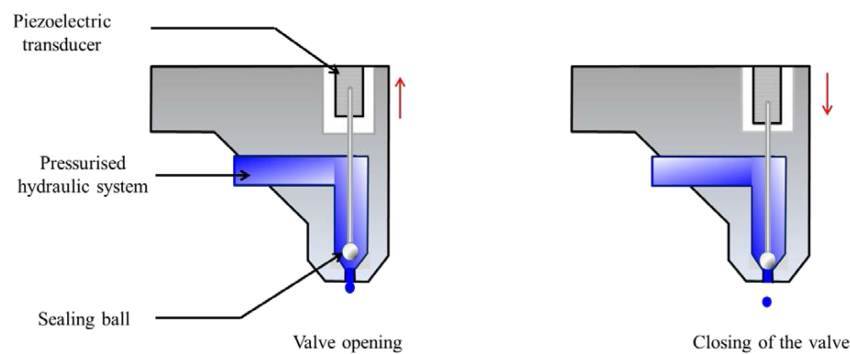

Closing of the valve

Figure 2. Diagram of operation of the generator opening valve.

system has been measured with the help of a Faraday pail connected to an electrometer (Keithley model 6514; Sow and Lemaitre, 2016). Any electrical charge on the drop was detected by our sensitive electrometer (limit of $10 \mathrm{fC}$ ). This might be explained by the fact that, unlike classical piezoelectric drop-on-demand systems (such as those of microdrop Technologies and MicroFab Technologies), the piezoelectric transducer in our drop generator is not in direct contact with the liquid (Fig. 2).

\subsubsection{Drop size measurements}

The generator was calibrated in order to produce drops of a prescribed diameter. Two parameters govern the size of the drops: the water supply pressure and the valve opening time. The different tests performed showed that when the pressure in the water circuit is too high, the drops break up at the injector outlet. Maintaining pressure below, or at 0.3 bar, avoids these effects. These tests were therefore performed at a positive pressure of 0.3 bar. For this water circuit supply pressure, the valve opening time was between 4 and $11 \mathrm{~ms}$. The raindrops' size is determined after a free-fall acceleration over a height of $8 \mathrm{~m}$. For each opening time, shadowgraph measurements were taken in the aerosol chamber of the BERGAME facility. An optical window is used to trigger the photographing of each drop entering the BERGAME aerosol chamber. Our optical device is a camera (Andor: neo, sCMOS) with a resolution of $2560 \times 2160$ square pixels. It is equipped with a Canon macro lens (MP-E $65 \mathrm{~mm}, \mathrm{f} / 2.8,1-5 \mathrm{x}$ ) for a magnification of $3: 1$ (experimentally checked with a calibration chart). The pixel size is $6.5 \mu \mathrm{m}$, for a spatial resolution of $2.1 \mu \mathrm{m}$. Drops are backlit with a $9 \mathrm{~ns}$ strobe to freeze their fall on the sensor. An example of a shadowgraph image is shown in Fig. 3.

Due to the oscillations, the millimetric drops exhibit an oblate spheroid shape. To define the size of the raindrops, the notion of "diameter equivalent to a sphere of the same volume" has been adopted. Since shadowgraphy yields only 2-D information, the diameters are equivalent to a disc. For axisymmetric objects, equivalent spherical diameter and equivalent disc diameter are equal. Szakál et al. (2009) experimentally verified this axial symmetry of the drop of that size range at terminal velocity. Thus, shadow images are used and

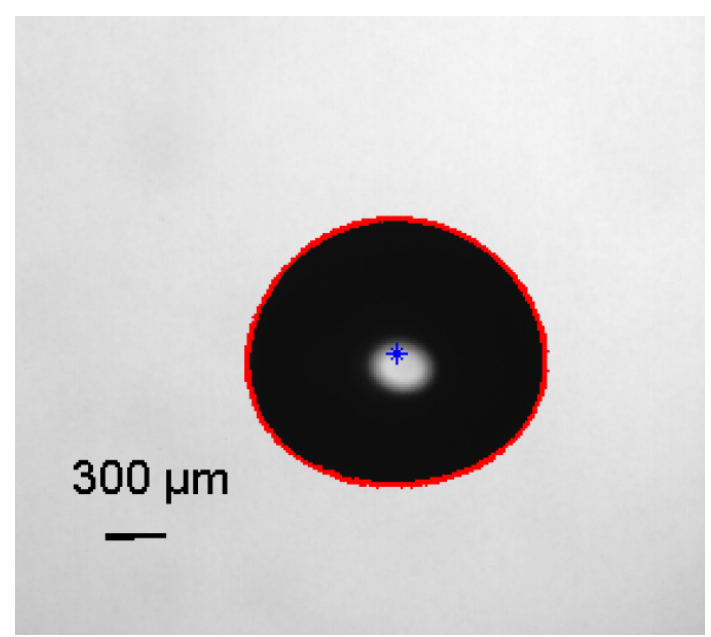

Figure 3. Example of a shadow image.

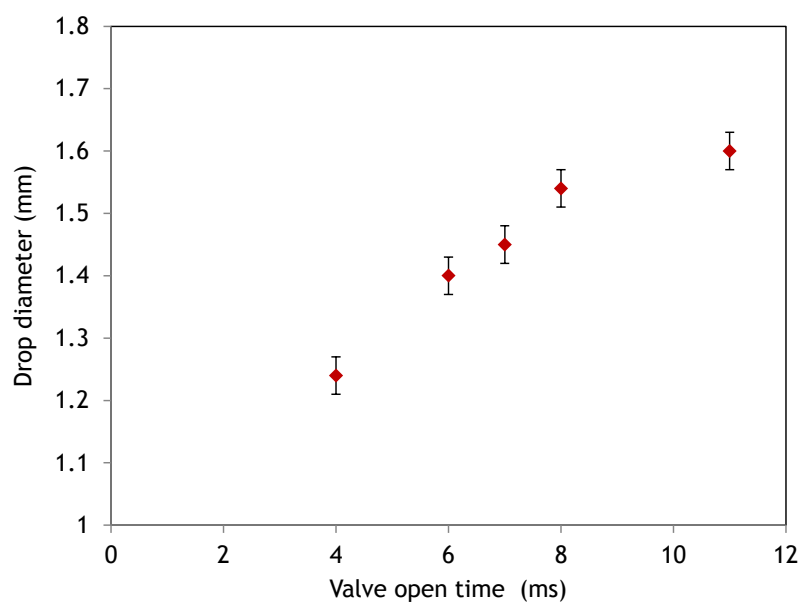

Figure 4. Measured equivalent diameter of the drop produced by our generator as a function of the valve opening time (for an overpressure of $0.3 \mathrm{bar}$ ).

processed to deduce the projected surface area of the drop $\left(S_{\text {drop }}\right)$ and derive the diameter of the disc of equal surface area $\left(D_{\text {eq }}\right)$.

$D_{\text {eq }}=\sqrt{\frac{4 S_{\text {drop }}}{\pi}}$

For each injection configuration, the equivalent diameter of the drops is measured for 100 images. Finally, the mean equivalent diameter and the standard deviation are calculated. Figure 4 shows all the measurement points investigated. For all operating points, the standard deviation is approximately $20 \mu \mathrm{m}$, i.e. approximately $1.5 \%$ of the size of the drop. 


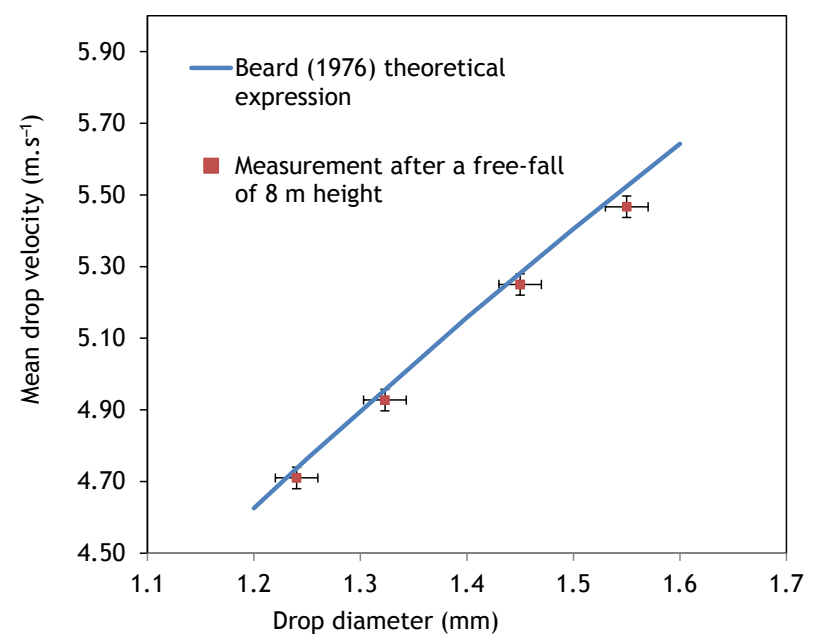

Figure 5. Comparison of velocities measured in BERGAME with the Beard (1976) model.

\subsubsection{Drop velocity measurements}

In order to be representative of rain, the drops must cross the BERGAME aerosol chamber at their terminal velocity. For each of the drop sizes produced by our generator, the drop fall velocity is also measured at the entrance of the aerosol chamber, below the $8 \mathrm{~m}$ free-fall shaft. Two consecutive pictures of the same drop are taken during its fall. By knowing the time interval between these two images and measuring the displacement of the centre of the drop, we derive its velocity. The results are shown in Fig. 5 and compared to the theoretical values computed from Beard (1976), often taken as the reference in the literature, as it was validated both in wind tunnel tests and in the environment.

We note in this figure that, up to a drop diameter of $1.4 \mathrm{~mm}$, the $8 \mathrm{~m}$ distance is sufficient for accelerating the drops to their terminal velocity. This is consistent with the results of the theoretical calculations of Wang and Pruppacher (1977b), which predict that $6.5 \mathrm{~m}$ free fall is enough for a $1.4 \mathrm{~mm}$ drop to reach $99 \%$ of terminal velocity. Furthermore, to ensure that our drops are representative of the hydrometeors described in the literature, we compare in Fig. 6 the axis ratios of the drops in the BERGAME chamber with the model of Beard and Chuang (1987). For the drop sizes investigated, the drop can be considered as horizontally aligned oblate spheroids (Fig. 3); no tilt angle was measured, which is consistent with Pruppacher and Beard (1970) measurements. This is why the axis ratio is computed as the ratio between the vertical and horizontal dimensions of the drop.

Figure 6 shows that, up to a diameter of $1.4 \mathrm{~mm}$, the drops entering the aerosol chamber are perfectly representative of the hydrometeors observed in the atmosphere.

In this study, we focus on the collection efficiency of drops with a diameter of $1.25 \mathrm{~mm}$. We have selected this size because it is the only one produced by our systems for which

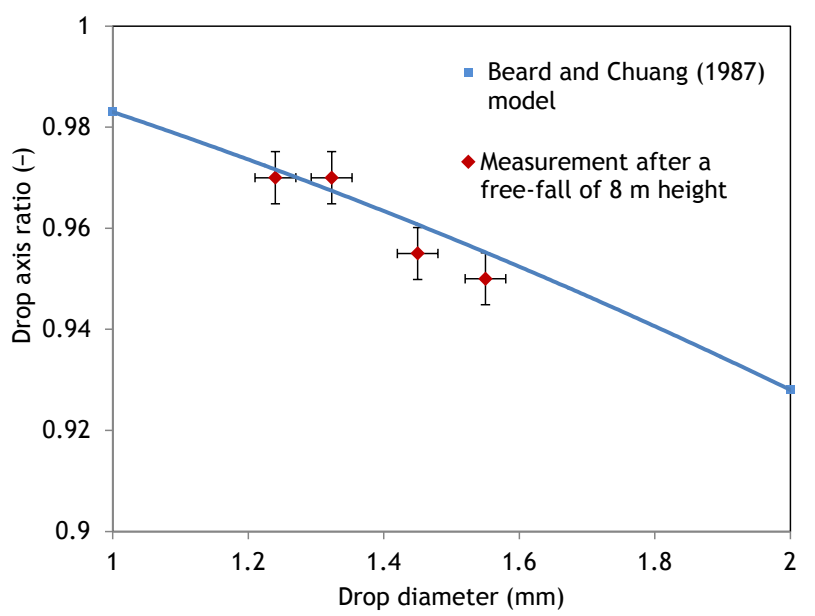

Figure 6. Comparison of axis ratios measured in BERGAME with the model of Beard and Chuang (1987).

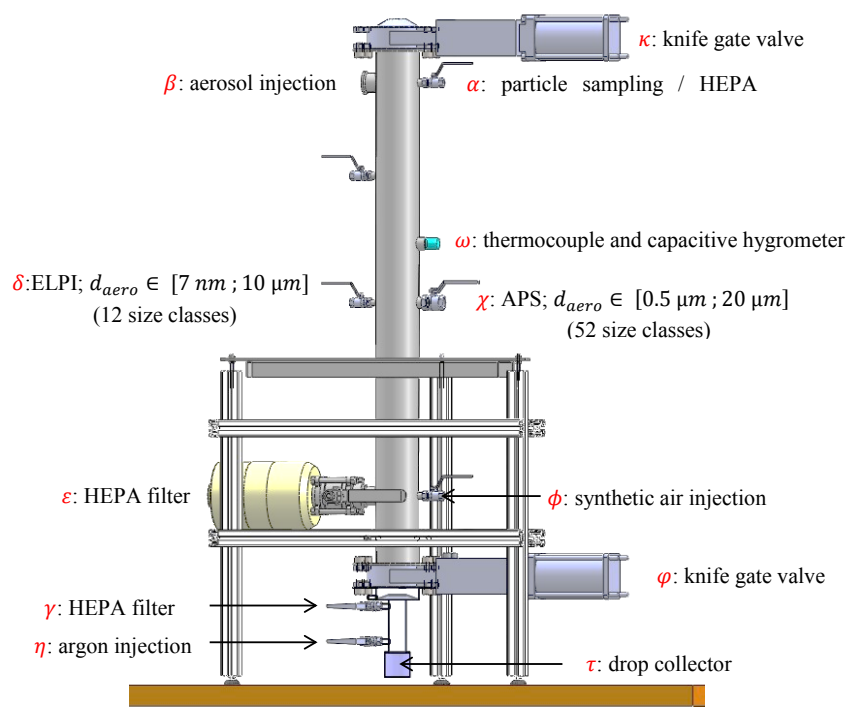

Figure 7. Schematic design of the new BERGAME aerosol chamber.

comparisons with Beard (1974) simulations will be direct. This model is particularly interesting as we have previously shown that, for $2 \mathrm{~mm}$ diameter drops (Quérel et al., 2014b), it is the only one able to predict the sharp rise in the collection efficiency observed experimentally for submicroscopic particles, which is due to the eddies that develop within the wake of the drop. These vortices will capture the particles and draw them back onto the rear of the drop. For a drop diameter of $1.25 \mathrm{~mm}$, an $8 \mathrm{~m}$ free-fall distance is enough for the drops to represent atmospheric raindrops, both in terms of velocity and axis ratio. 

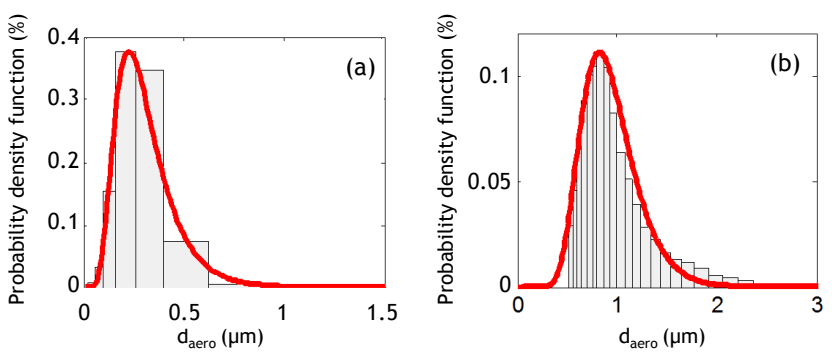

Figure 8. The size distribution of the particles produced by the ultrasound aerosol generator vibrating at $2400 \mathrm{kHz}$ (a) for a fluorescein concentration of $0.11 \mathrm{~g} \mathrm{~L}^{-1}$ and (b) for a fluorescein concentration of $10 \mathrm{~g} \mathrm{~L}^{-1}$. The distribution on the left is measured using an electrical low-pressure impactor (ELPI, $\delta$ ) and the one on the right using an aerodynamic particle sizer (APS, $\chi$ ).

\subsection{Description of the new BERGAME aerosol chamber}

A new aerosol chamber (Fig. 7) has been designed to increase the concentration of particles within the volume swept by the drops during their fall. Its geometry is strongly influenced by the one developed by Hampl et al. (1971). It consists of a $1300 \mathrm{~mm}$ high stainless steel cylinder with an internal diameter of $100 \mathrm{~mm}$.

Various taps are provided for injecting the aerosols, taking samples and characterising the thermodynamic conditions of the gas. These various sampling points serve to measure, in particular,

- the aerosol particles' size distribution,

- their mass concentration and

- the temperature and relative humidity.

In Fig. 7, each valve is labelled with a Greek letter to structure the explanations in the text. The chamber is fitted with two gate valves, one at the top $(\kappa)$ and the other at the bottom $(\varphi)$. These two valves isolate the chamber while it is being filled with particles. The particle size distribution of the aerosols is measured by means of an aerodynamic particle sizer (APS, $\chi$ ) and an electrical low-pressure impactor (ELPI, $\delta$ ). The injected particles are pure fluorescein particles so that they may be easily measured by fluorescence spectrometry. The mass concentration of the particles in suspension inside the chamber is determined by venting the entire content of the chamber onto a high-efficiency particulate arresting (HEPA) filter $(\alpha)$ and measuring the mass of particles on the filter using fluorescence spectrometry.

Finally, the relative humidity and the temperature are given, respectively, by a capacitive hygrometer and a thermocouple $(\omega)$.

After having accelerated in free fall over $8 \mathrm{~m}$, the drops are representative of rain in terms of size, velocity and axis ratio (Sect. 2.1). They enter the aerosol chamber via a circular opening with a $4 \mathrm{~cm}$ diameter. After crossing the aerosol chamber, the drops are collected in a removable container $(\tau)$. One of the principal difficulties of these experiments relates to the sedimentation of the cloud of particles that settles directly inside the drop collector. Indeed, RayleighTaylor instabilities can arise when a dense cloud of aerosol particles overlies a layer of clean air. These instabilities induce a downward motion of the aerosol cloud much faster that the settling velocity of individual particles (Hinds et al., 2002). In order to avoid this effect, a layer of argon (which is denser than the cloud of particles) is formed in the bottom of the aerosol chamber, located below the second gate valve in Fig. 7. A large number of experiments was performed. These experiments show that, regardless of the concentration and the size of the particles in the aerosol chamber, until $4 \mathrm{~min}$ after opening the gate valves, the drop collector is free from any particulate contamination. Beyond $4 \mathrm{~min}$, traces of fluorescein are detected on the drop collector.

\subsection{Aerosol particle characterisation and generation}

The aerosol particles size distributions are measured using an $\operatorname{ELPI}(\delta)$ and an APS $(\chi)$.

ELPI is a quasi-real-time aerosol spectrometer (Marjamäki et al., 2000). It is composed of a corona charger and a 12-stage cascade low-pressure impactor. Each stage of the impactor is connected to an electrometer. The corona charger is used to set the electrical charge of the particles to a specific level. Then, the low-pressure impactor classifies the aerosol particles into 12 size classes according to their aerodynamic diameter (from $7 \mathrm{~nm}$ to $10 \mu \mathrm{m}$ ). Finally, the electrometers measure the electrical charge carried by the particles collected by each impaction stage. This charge is finally converted to the number of particles collected according to the charging efficiency function of the corona charger.

APS is also a quasi-real-time aerosol spectrometer (Baron, 1986). It measures the time of flight of individual particles accelerated by a controlled accelerating flow imposed by a calibrated nozzle. The time of flight of each aerosol particle is then converted into its aerodynamic diameter. Thus, the APS classifies the aerosol particles in terms of aerodynamic diameter from $500 \mathrm{~nm}$ to $20 \mu \mathrm{m}$ over 52 size classes.

APS and ELPI are both used for their complementary size ranges so all the particles produced in our laboratory can be sized. For particles with a median aerodynamic diameter less than $0.8 \mu \mathrm{m}$, the size distribution is measured using an ELPI. For the others, we favour the use of an APS because of the better size resolution.

The aerosol particles are produced with two ultrasound generators. The key part of these generators is a piezoelectric ceramic immersed in a solution. When subjected to an appropriate electric current, this ceramic vibrates at a frequency of 500 or $2400 \mathrm{kHz}$ depending on the generator used. 
These oscillations transform the surface of the liquid into a mist of microscopic droplets with a narrow size distribution. These drops are transported to the upper part of the generator by a flow of dry filtered air at a flow rate of $20 \mathrm{~L} \mathrm{~min}^{-1}$. More dry air is added in the upper part of the generator at a flow rate of $30 \mathrm{~L} \mathrm{~min}^{-1}$ to dry the particles.

These drying and dispersal flow rates have been selected to obtain the following characteristics:

- the aerosol particle size distributions are narrowly spread (geometric standard deviation less than or equal to 1.5$)$;

- the particle concentration inside the aerosol chamber is high $\left(\sim 2 \times 10^{5}\right.$ particles $\left.\mathrm{cm}^{-3}\right)$; and

- the relative humidity measured in the aerosol chamber is approximately $77 \pm 1 \%$. This humidity corresponds to relative humidities observed during rainfall events (Depuydt et al., 2012). Furthermore, we will show that this humidity is high enough to make diffusiophoresis negligible (see the discussion of Fig. 12 in Sect. 3).

Changing the concentration of the solute dissolved in the water varies the size of the produced particles. The chosen solute is sodium fluorescein $\left(\mathrm{C}_{10} \mathrm{H}_{10} \mathrm{Na}_{2} \mathrm{O}_{5}\right)$. This molecule has been selected for its very large fluorescence properties. It can be easily detected by fluorescence spectroscopy down to a concentration of $5 \times 10^{-11} \mathrm{~g} \mathrm{~mL}^{-1}$. The generator is placed inside a negative pressure enclosure to prevent any possible fluorescein particle contamination of the laboratory. Figure 8 shows two examples of number particle size distributions of fluorescein measured in the BERGAME aerosol chamber.

Both of these distributions fit well to log-normal distributions (red curves on the graphs). For a fluorescein concentration of $0.11 \mathrm{~g} \mathrm{~L}^{-1}$ (respectively, $10 \mathrm{~g} \mathrm{~L}^{-1}$ ) in the solution, the median diameter of the fitted distribution is $220 \mathrm{~nm}$ (respectively, $820 \mathrm{~nm}$ ) and the geometric standard deviation is 1.5 (respectively, 1.34).

For each of the particle sizes produced, the fluorescein mass concentrations in the aerosol chamber derived from APS and ELPI measurements are compared with ones derived from filter measurements (Sect. 2.2). These comparisons provide slight differences $(\sim 10 \%)$ that can be attributed to both the purity of fluorescein sodium salt used $(\sim 97 \%)$ and the shape of the aerosol particles that is not perfectly spherical. Thus, for improving the accuracy of collection efficiency measurements, the fluorescein concentration inside the aerosol chamber is derived from filter measurements, and APS and ELPI are used to provide a precise measurement of the particle size.

In order to neutralise the charge of the aerosol particles prior to injecting them into the BERGAME aerosol chamber $(\beta)$, the particles go through a low-energy X-ray neutraliser $\left(<9.5 \mathrm{keV}\right.$, TSI 3088), at a flow rate of $1.5 \mathrm{~L} \mathrm{~min}^{-1}$. At this flow rate, the residence time of the particles in the neutraliser is sufficient to neutralise them.
As we have seen in the previous section, our aerosol generator produces aerosols at a flow rate of $50 \mathrm{~L} \mathrm{~min}^{-1}$ (20 L min ${ }^{-1}$ of dispersion air and $30 \mathrm{~L} \mathrm{~min}^{-1}$ of drying air). Therefore, we use a flow divider to ensure that the particles pass through the neutraliser at $1.5 \mathrm{~L} \mathrm{~min}^{-1}$. This divider includes an $8 \mathrm{~L}$ buffer volume, provided with one inlet and two outlets. A flow rate of $48.5 \mathrm{Lmin}^{-1}$ is drawn off from one of these outlets by means of an air suction pump. This flow is filtered and vented. The remaining flow passes through the neutraliser. After neutralisation, the particles are injected into the aerosol chamber.

\subsection{Test procedure}

The aerosol chamber is flushed at the start of each experiment with synthetic air to ensure that initial conditions are free of any fluorescein particle contamination. After flushing, the previously neutralised aerosol particles of chosen diameter are injected at a flow rate of $1.5 \mathrm{~L} \mathrm{~min}^{-1}$ via valve $\beta$ (Sect. 2.3).

The two knife gate valves ( $\varphi$ and $\kappa$ ) are closed during this filling phase in order to isolate the enclosure. In addition, valve $\varepsilon$ is opened to exhaust the excess pressure towards a HEPA filter. The injection process lasts $20 \mathrm{~min}$, during which we form a layer of argon within the zone located below knife gate valve $\phi$. This injection is carried out in two stages. First, we inject the argon during 10 min via valve $\eta$, with the drop collector unscrewed and valve $\gamma$ closed. Second, the drop collector is refitted and valve $\gamma$ is opened. At the end of this phase, the aerosol chamber is filled with neutralised particles of a prescribed diameter at a concentration of approximately $2 \times 10^{5}$ particles per cubic centimetre.

This filling phase of the enclosure is followed by a relaxation period lasting no less than $15 \mathrm{~min}$. During this time period, all the valves of the aerosol chamber are closed, with the exception of valve $\varepsilon$, which remains open in order to perfectly balance the pressures. This period is used to bring the train of drops produced by the generator to the centre line of the aerosol chamber. Once the drop generator is adjusted, valve $\varepsilon$ is closed and both knife gate valves $(\varphi$ and $\kappa)$ are opened to enable the drops to cross the aerosol chamber. A cumulated volume of $1 \mathrm{~cm}^{3}$ of solution is necessary for performing a measurement by fluorescence spectrometry, i.e. approximately 1000 drops of $1.25 \mathrm{~mm}$ diameter. As a result of the frequency at which drops cross the enclosure, $10 \mathrm{~min}$ are needed to collect this volume. As mentioned above, the drop collector remains free of any particulate contamination if the valves remain open for less than $4 \mathrm{~min}$. The $10 \mathrm{~min}$ needed to collect the 1000 drops are therefore divided into three periods of $200 \mathrm{~s}$ each. At the end of these $200 \mathrm{~s}$ phases, the gate valves are closed again and the buffer volume between gate valve $\phi$ and the drop collector is flushed with argon (Fig. 9). During flushing, the argon is injected through valve $\eta$ and removed through valve $\gamma$, which ensures an up- 
ward flow within this buffer volume and minimises the risk of contamination of the drop collector.

Once $1 \mathrm{~cm}^{3}$ of drops is collected, both knife gate valves close, and the buffer volume is flushed, to avoid any contamination of the collected water when removing the drop collector.

In order to determine the collection efficiency, we need to know the mass concentration of fluorescein within the volume swept by the drops (Eq. 3). The concentration is measured by filter analysis, and for this purpose the aerosol chamber of the BERGAME experiment is flushed with synthetic air at the end of each experiment. This is done by injecting the synthetic air through valve $\varphi$, at a flow rate of $5 \mathrm{~L} \mathrm{~min}^{-1}$ for $10 \mathrm{~min}$, and collecting the particles on a HEPA filter.

This filter is then placed in $100 \mathrm{~mL}$ of ammonia water $\left(V_{\text {sol }}\right)$ for $24 \mathrm{~h}$ in order to dissolve all the fluorescein particles it contains. Finally, the mass concentration of fluorescein in this water $\left([\text { fluo }]_{\text {filter }}\right)$ is measured by fluorescence spectrometry.

The mass concentration of fluorescein particles in the aerosol chamber ([fluo $]_{\text {chamber }}$ ) is then determined using the following equation:

$[\text { fluo }]_{\text {chamber }}=\frac{[\text { fluo }]_{\text {filter }} \cdot V_{\text {sol }}}{V_{\text {chamber }}}$.

In this equation, the term $V_{\text {chamber }}$ is the volume of the aerosol chamber, i.e. $10.2 \mathrm{~L}$.

As the mass concentration of particles is only quantified once the measurements are completed, we have attempted to quantify its variation over the duration of a measurement (approximately $15 \mathrm{~min}$ ). For this, we have first verified the reproducibility of characteristics of the aerosol produced by the aerosol generator in size, number and concentration. This is performed by repeating the injection phase with exactly the same operating conditions. No variation of the fluorescein concentration greater than the uncertainty of the fluorimeter ( $\pm 2.5 \%$; Appendix A) has ever been measured. We have then compared the mass concentration in the aerosol chamber just after the relaxation phase and after a complete measurement procedure. At last, we measured a reduction in concentration of less than $8 \%$ regardless of the particle diameter. These particles are essentially lost through deposition on the sides of the aerosol chamber. This decrease of the particle concentration during the experiments is the main source of uncertainty on the measurement of the collection efficiency.

The collection efficiency is defined as the ratio between the mass of particles (of a given diameter) collected by a drop as it falls and the total mass of particles (of the same diameter) within the volume it has swept. The mass of fluorescein in the drops during the experiments $\left(M_{\text {drop }}\right)$ is easy to calculate:

$M_{\mathrm{drop}}=\frac{\pi D_{\mathrm{drop}}^{3}}{6}[\text { fluo }]_{\mathrm{drop}}$,

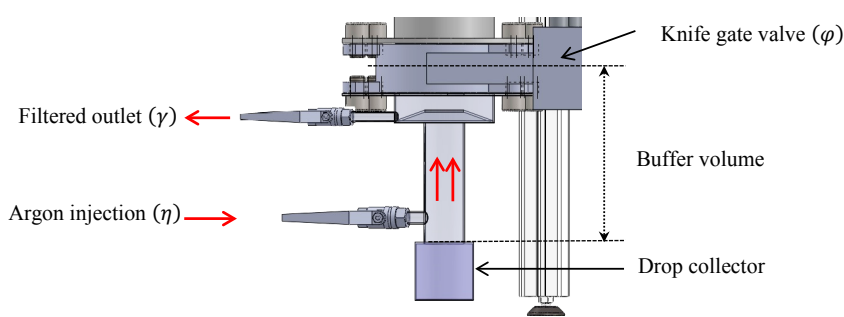

Figure 9. Buffer volume flushing procedure.

where $[\text { fluo }]_{\text {drop }}$ is the mass concentration of fluorescein in the drops.

The mass of particles within the volume swept by the drops $\left(M_{2}\right)$ is calculated with

$M_{2}=\frac{\pi D_{\text {drop }}^{2} H}{4}[\text { fluo }]_{\text {chamber }}$,

where [fluo $]_{\text {chamber }}$ is the mass concentration of fluorescein in the aerosol chamber and $H$ the height of the aerosol chamber (1.3 m; Fig. 1).

The collection efficiency is derived from the following expression:

$E\left(d_{\text {aero }}, D_{\text {drop }}, \mathrm{RH}\right)=\frac{M_{\text {drop }}}{M_{2}}=\frac{2 D_{\text {drop }} \cdot[\text { fluo }]_{\text {drop }}}{3 H \cdot[\text { fluo }]_{\text {chamber }}}$.

In order to precisely determine the size distribution of the particles for which the collection efficiency has been measured, we repeat the injection of particles into the BERGAME aerosol chamber following each efficiency measurement under exactly the same operating conditions (generator, ceramic excitation frequencies, injection times, dispersal and drying flow rates and fluorescein concentration). The size distribution of the aerosol particles produced by the generator is then measured in the aerosol chamber.

\section{Results and discussion}

All the measurements taken are summarised in Table 2 with the associated expanded relative uncertainties. The first column of this table provides the median aerodynamic diameter $\left(d_{\text {aero }}\right)$ of each particle size distribution measured using the APS or the ELPI. The detailed calculation of the uncertainties is presented in Appendix A1 (Lira, 2002).

This median aerodynamic diameter is converted into a physical diameter $\left(d_{\mathrm{ap}}\right)$ by means of the following expression (which is solved iteratively):

$d_{\mathrm{ap}}=d_{\mathrm{aero}} \sqrt{\frac{C_{\mathrm{c}, d_{\mathrm{aero}}}}{C_{\mathrm{c}, d_{\mathrm{ap}}}}\left(\frac{\rho_{0}}{\rho_{\mathrm{p}}}\right)}$.

In this equation, $C_{\mathrm{c}}$ is the Cunningham slip correction factor and $\rho_{0}$ the water density. The density of the particle $\left(\rho_{\mathrm{p}}\right)$ 
Table 2. Summary of measurements performed.

\begin{tabular}{lcrccc}
\hline $\begin{array}{l}d_{\text {aero }} \\
(\mu \mathrm{m})\end{array}$ & $\begin{array}{r}D_{\text {drop }} \\
(\mathrm{mm})\end{array}$ & $\begin{array}{r}\text { RH } \\
(\%)\end{array}$ & $\begin{array}{c}{[\text { fluo }]_{\text {drop }}} \\
\left(\mathrm{g} \mathrm{cm}^{-3}\right)\end{array}$ & $\begin{array}{c}{[\text { fluo }]_{\text {chamber }}} \\
\left(\mathrm{g} \mathrm{cm}^{-3}\right)\end{array}$ & $\begin{array}{c}E \\
(-)\end{array}$ \\
\hline 0.25 & & & $8.22 \times 10^{-8} \pm 2.5 \%$ & $6.22 \times 10^{-9} \pm 8 \%$ & $8.8 \times 10^{-3} \pm 16 \%$ \\
0.25 & & & $1.15 \times 10^{-7} \pm 2.5 \%$ & $7.91 \times 10^{-9} \pm 8 \%$ & $9.7 \times 10^{-3} \pm 16 \%$ \\
0.5 & & & $3.39 \times 10^{-8} \pm 2.5 \%$ & $4.22 \times 10^{-9} \pm 8 \%$ & $5.4 \times 10^{-3} \pm 16 \%$ \\
0.6 & $1.25 \pm 1.5 \%$ & & $4.51 \times 10^{-8} \pm 2.5 \%$ & $1.38 \times 10^{-8} \pm 8 \%$ & $2.2 \times 10^{-3} \pm 16 \%$ \\
0.71 & & $77 \pm 5 \%$ & $2.15 \times 10^{-8} \pm 2.5 \%$ & $9.62 \times 10^{-9} \pm 8 \%$ & $1.5 \times 10^{-3} \pm 16 \%$ \\
1 & & & $2.52 \times 10^{-7} \pm 2.5 \%$ & $1.17 \times 10^{-9} \pm 8 \%$ & $2.9 \times 10^{-3} \pm 16 \%$ \\
1.47 & & $5.48 \times 10^{-8} \pm 2.5 \%$ & $6.39 \times 10^{-9} \pm 8 \%$ & $5.7 \times 10^{-3} \pm 16 \%$ \\
2.54 & & $6.51 \times 10^{-7} \pm 2.5 \%$ & $5.51 \times 10^{-9}, \pm 8 \%$ & $7.9 \times 10^{-2} \pm 16 \%$ \\
\hline
\end{tabular}

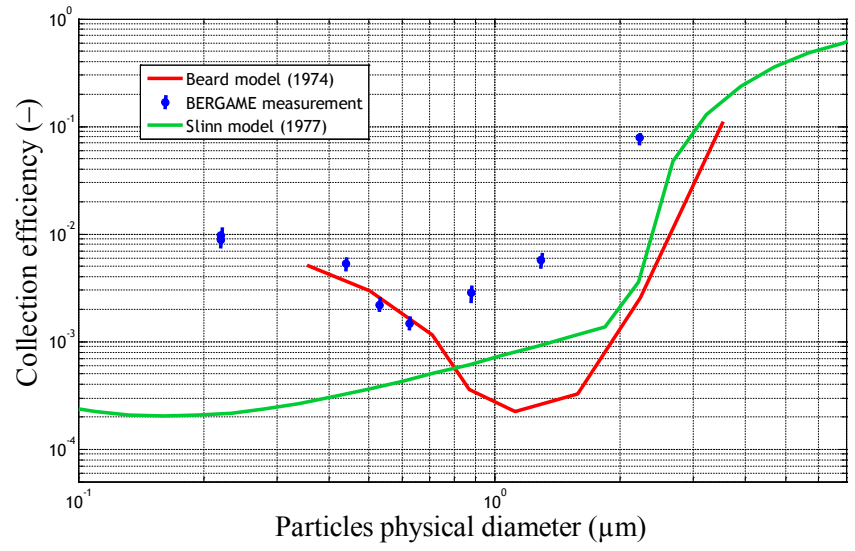

Figure 10. Comparison of our measurements for a drop of $1.25 \mathrm{~mm}$ diameter with the results of the models of Beard (1974) and Slinn (1977).

is calculated from the growth factor (GF) of the fluorescein aerosol particle.

$\rho_{\mathrm{p}}=\frac{\rho_{\mathrm{C}_{10} \mathrm{H}_{10} \mathrm{Na}_{2} O_{5}}+\rho_{0}\left(\mathrm{GF}^{3}-1\right)}{\mathrm{GF}^{3}}$

This factor has previously been measured using a hygroscopic tandem differential mobility analyser (HTDMA; Quérel et al., 2014b). For our experiments, performed at a relative humidity of $77 \pm 5 \%$, we deduce a GF of $1.25 \pm 0.05$. Stöber and Flachsbart (1973) have measured a density of $1.58 \mathrm{~g} \mathrm{~cm}^{-3}$ for a dry fluorescein aerosol particle. Using Eq. (10), we therefore calculate the density of our aerosol in the aerosol chamber to be $1.30 \pm 0.05 \mathrm{~g} \mathrm{~cm}^{-3}$.

The aerodynamic diameters measured in the aerosol chamber by the APS and the ELPI can then be expressed as physical diameters $\left(d_{\mathrm{ap}}\right)$ :

$d_{\mathrm{ap}}=0.88 \times d_{\text {aero }}$.

All our measurements are summarised in Table 2 and plotted in Fig. 10 as a function of the median diameter of the distribution of the physical diameter of the particles. Figure 10 compares our dataset against the efficiencies computed by both Slinn (1977) and Beard (1974) models. In this figure, the Slinn model includes the contributions of inertial impaction, Brownian diffusion and interception (Table 1). It should be remembered that the in situ scavenging measurements (Volken and Shumann, 1993; Laakso et al., 2003; Chate, 2005) are only compared to the Slinn model. In the aerosol size range investigated, the collection efficiencies measured vary considerably as a function of the particle size. On a logarithmic scale, the efficiency curve obtained has a "V" shape with a minimum around $0.65 \mu \mathrm{m}$. The increase in the collection efficiency for particles larger than $0.65 \mu \mathrm{m}$ is attributed to the mechanism of impaction on the front side of the drop. Within this size range, the increase in the diameter of the particle increases its inertia. The particle can then no longer follow the streamlines and impacts the drop.

The reasons for the increase in the collection efficiency for particles smaller than $0.65 \mu \mathrm{m}$ in diameter are not as easy to figure out. Indeed, particles of this size range are not expected to be affected by Brownian motion since their diameter is 7 times bigger than the mean free path of the air molecules.

The Slinn model does not predict this increase and underestimates the collection efficiency for a $0.22 \mu \mathrm{m}$ particle by 2 orders of magnitude. This is linked to the assumptions of Stokes flow around the drop. Yet, at Reynolds numbers larger than 20 (for a $280 \mu \mathrm{m}$ drop at its terminal velocity), recirculation eddies develop in the wake of the drop. Beard (1974) has shown the major influence of these wake vortices on the collection of submicron-sized particles. In fact, he showed that the smallest aerosol particles are trapped in these eddies in the wake of the drop and then collected on its rear side.

This model is not referred to in the literature, as it has never been validated by experiments until now. Yet, we observe that, for particles below this minimum efficiency, our measurements are in almost perfect agreement with the model and seem to validate it.

For particles with a diameter greater than $1 \mu \mathrm{m}$, we observe that the Beard or Slinn models yielded almost the same val- 
ues. This result is expected since their only difference stands in the Stokes flow around the drop for Slinn model. This assumption prevents the capture of boundary layer separation in the wake of the drop and the resulting recirculating flows even if it makes very little difference to the flow on the leading edge of the drop. Yet, particles with a diameter greater than $1 \mu \mathrm{m}$ are very sensitive to inertial effects and are captured on this front side. Moreover, as the Stokes number of these large particles is high, they pass through the recirculations without being trapped.

For particles with a diameter greater than $0.65 \mu \mathrm{m}$, our measurements show the same trends as these two models but with an average difference of 1 order of magnitude. This is probably related to the fact that, during our experiments, the aerosol particles in the aerosol chamber are not perfectly mono-disperse. Indeed, the particles have log-normal distributions with geometric standard deviations between 1.3 and 1.5 (Fig. 8). The collection efficiency varies very sharply with particle size. Thus, in order to compare more rigorously our measurements with the Beard (1974) model, we need to calculate, for each measurement, the average theoretical collection efficiency $\left(\left\langle E\left(D_{\mathrm{gtte}}, d_{\mathrm{ap}}\right)\right\rangle\right)$ resulting from the integration of the Beard (1974) model over the entire range of particle sizes in the aerosol chamber (Eq. 12).

$$
\begin{aligned}
& \left\langle E\left(D_{\mathrm{drop}}=1.25 \mathrm{~mm}, d_{\mathrm{ap}}\right)\right\rangle \\
& =\frac{\int_{d_{\mathrm{ap}=0}}^{\infty} f\left(d_{\mathrm{ap}}\right) \cdot d_{\mathrm{ap}}^{3} E\left(D_{\mathrm{drop}}=1.25 \mathrm{~mm}, d_{\mathrm{ap}}\right) \mathrm{d} d_{\mathrm{ap}}}{\int_{d_{\mathrm{ap}=0}}^{\infty} f\left(d_{\mathrm{ap}}\right) \cdot d_{\mathrm{ap}}^{3} \cdot \mathrm{d} d_{\mathrm{ap}}}
\end{aligned}
$$

In this equation, the term $f\left(d_{\mathrm{ap}}\right)$ is the probability density function according to the number of the particles in the BERGAME aerosol chamber, and $E\left(D_{\text {drop }}=\right.$ $\left.1.25 \mathrm{~mm} d_{\mathrm{ap}}\right)$ is the collection efficiency calculated by the Beard model (1974) for a drop $1.25 \mathrm{~mm}$ in diameter. The numerator and denominator of this equation are both weighted by a term $d_{\mathrm{ap}}^{3}$, which reflects the fact that, experimentally, we measure intensities of fluorescence and therefore masses of particles. We use the rectangle method to numerically solve this integral. In addition, the functions $E\left(D_{\text {drop }}=\right.$ $\left.1.25 \mathrm{~mm} d_{\mathrm{ap}}\right)$ and $f\left(d_{\mathrm{ap}}\right)$ are both interpolated using Hermite interpolation polynomials (Fritsch and Carlson, 1980) with a step size of $0.1 \mu \mathrm{m}$.

We note a significant improvement of the agreement between our measurements and the Beard (1974) model since it is integrated over the entire particle size distribution measured during our experiments in BERGAME (red dots in Fig. 11). Larger differences are nevertheless observed for the first $\left(d_{\mathrm{ap}}=0.22 \mu \mathrm{m}\right)$ and last measurement points $\left(d_{\mathrm{ap}}=\right.$ $2.54 \mu \mathrm{m})$. These differences could be attributed to the fact that, for these points, the resolution of Eq. (12) requires an extrapolation of Beard (1974) calculations beyond the size range he investigated (continuous line in Fig. 11).

Moreover, for the collection efficiency measured for the finest aerosol particles $\left(d_{\mathrm{ap}}=0.22 \mu \mathrm{m}\right)$, the discrepancy ob- served with the Beard model could also be explained by the hypothesis of the simulations. Indeed, the Brownian motion was neglected. This can be justified in the particle size range investigated; however, it is much less justified when extrapolating the simulations to finer aerosol particles.

Furthermore, it is interesting to compare our measurements with the ones from Lai et al. (1978) since they are the only ones in the literature in the same drop size range. As the aerosol particles produced in these experiments are composed of silver chloride $\left(\rho_{\mathrm{AgCl}}=5.6 \mathrm{~g} \mathrm{~cm}^{-3}\right)$, which is much

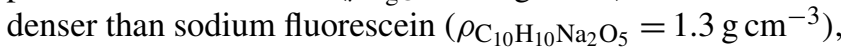
it is more appropriate to plot all the collection efficiencies as a function of the Stokes number of the particle $\left(S t_{\mathrm{ap}}\right)$.

$S t_{\mathrm{ap}}=\frac{\rho_{\mathrm{p}} U_{\infty}\left(D_{\mathrm{drop}}\right) d_{\mathrm{ap}}^{2} C_{\mathrm{c}, d_{\mathrm{ap}}}}{9 D_{\mathrm{drop}} \mu_{\mathrm{air}}}$

In this equation, $\mu_{\text {air }}$ is the dynamic viscosity of the air and $\rho_{p}$ the density of the aerosol particles. This comparison is presented in Fig. 12.

For particles with a Stokes number greater than $6 \times 10^{-2}$, the motion of the particles is driven by their inertia, leading us to expect to observe the same trends in our measurement and those of Lai et al. (1978). The comparison for a Stokes number smaller than $6 \times 10^{-2}$ is much less obvious. Indeed, for these particles, the measurements of Lai et al. (1978) indicate an increase in the collection efficiency, while our measurements continue to decrease down to a Stokes number of $1.6 \times 10^{-2}$. At that point, the slopes of the increases of both collection efficiency measurements are similar, while the Stokes number decreases.

A precise analysis of the procedure for the aerosol particle injection in the experiments of Lai et al. (1978) indicates that the carrier gas is pure nitrogen without any subsequent humidification. As a consequence, it is reasonable to consider that their measurements were performed with $0 \%$ relative humidity. In order to compare the contribution of diffusiophoresis for both our experiment and that of Lai et al. (1978), we plot in Fig. 12 the elementary contribution of diffusiophoresis $\left(E_{\mathrm{dph}}\right)$ to the collection efficiency. This contribution is calculated with the Davenport and Peters (1978) model for $0 \%$ relative humidity (as expected for the experiments of Lai et al., 1978) and $77 \%$ (as measured in our experiments). From this figure, it will be noted that, for the experiments of Lai et al. (1978), the contribution of diffusiophoresis is more than 1 order of magnitude higher than in ours. Furthermore, while in our experiments the contribution of diffusiophoresis is smaller than the collection efficiency simulated by Beard (1974), the opposite is observed with Lai et al. (1978). Thus, it appears that the experiments of Lai et al. (1978) cannot be compared directly to the model of Beard (1974), because they seem to be dominated by diffusiophoresis.

Based on these comparisons, we can consider that the Beard (1974) model is validated for addressing the collec- 


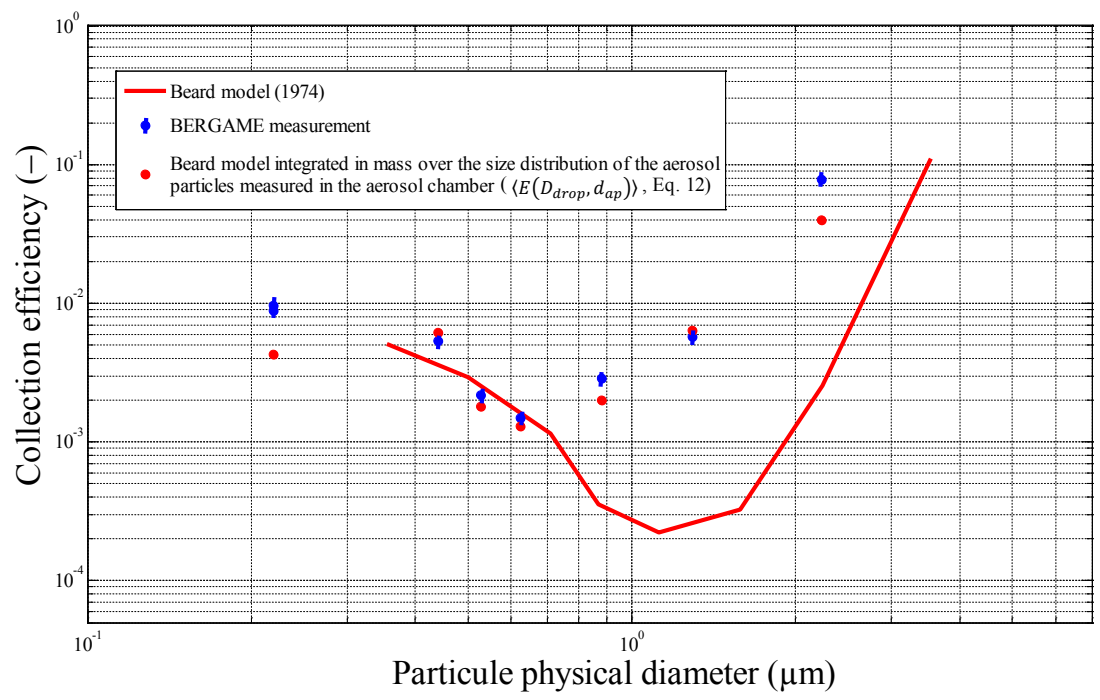

Figure 11. Integration of the Beard (1974) model over the particle size distribution of each of our experiments for a drop of $1.25 \mathrm{~mm}$ diameter.

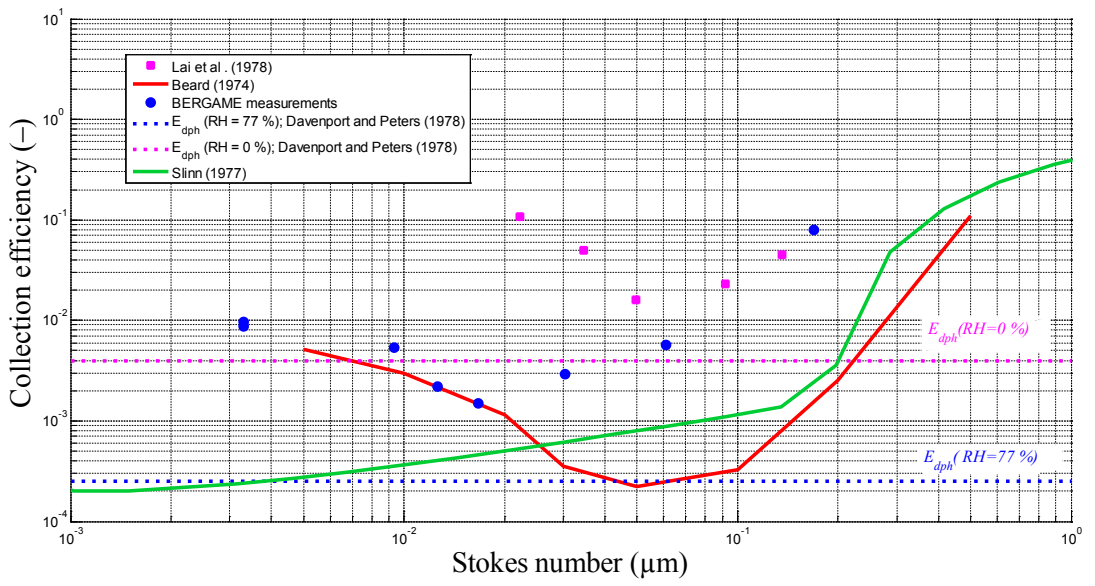

Figure 12. Collection efficiencies measured in this study and by Lai et al. (1978). Both measurements are compared to the Slinn (1977) and Beard (1974) models. The contribution of diffusiophoresis in both studies is computed following the description of Davenport and Peters (1978).

tion of the aerosol particles of the accumulation mode by raindrops. Finally, it seems necessary to provide, to facilitate its use, an analytical expression to assess the contribution of the rear capture ( $\left.E_{\mathrm{Re}-c a p t u r e}\right)$ to the raindrop collection efficiency. Indeed, the Slinn (1977) model which neglects rear capture underestimates the collection efficiency by 2 orders of magnitude in the submicron range compared to Beard's model (1974). Furthermore, Beard (1974) noticed from his theoretical simulations that rear capture plays a main role in the collection efficiency for aerosol particles with a Stokes number smaller than $5 \times 10^{-2}$. Thus, to derive an analytical expression for the elementary collection efficiency resulting from rear capture alone $\left(E_{\text {rear capture }}\right)$, we gather in Fig. 13 the collection efficiencies numerically simulated by Beard (1974) for a Stokes number smaller than $5 \times 10^{-2}$ (crosses in Fig. 13). These collection efficiencies are plotted as a function of the Reynolds number of the drops and the Stokes number of the particles.

This figure suggests that the Reynolds number of the drop and Stokes number of the aerosol particles are the two parameters influencing rear capture. The dependency on these two dimensionless numbers is physical, as the Reynolds number of the drop $\left(R e_{\text {drop }}\right)$ reflects the intensity and the size of the areas of recirculating flow in its wake and the particle Stokes number $\left(S t_{\mathrm{ap}}\right)$ reflects the susceptibility of the particle to pass through the recirculating flow in the wake of the drop without being trapped. 


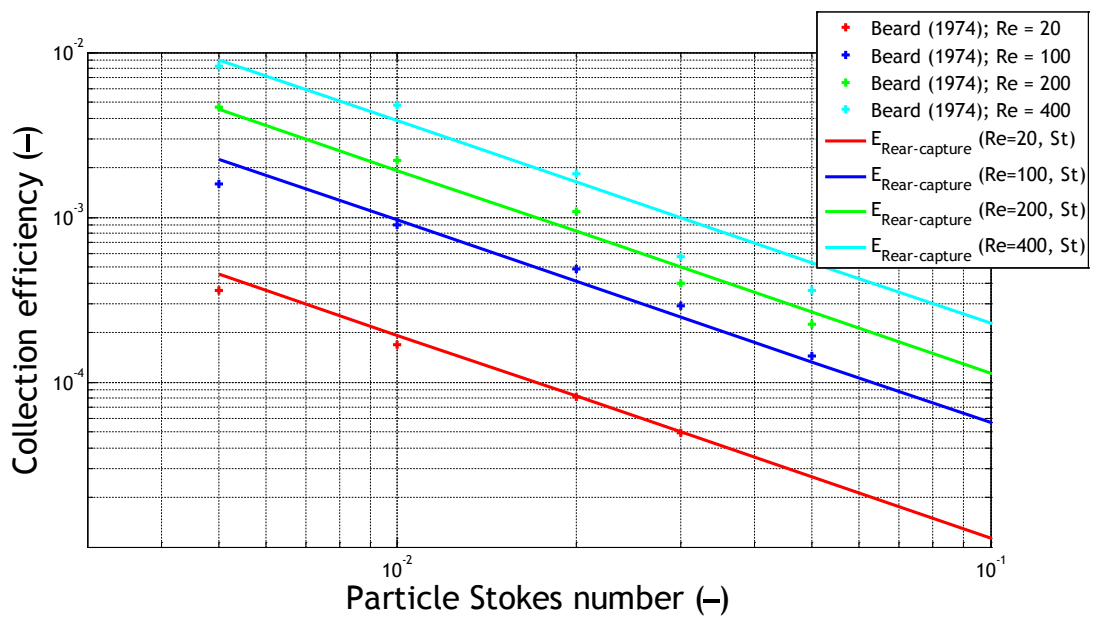

Figure 13. Semi-empirical parameterisation of rear capture.

Applying a power law fit to the simulations of Beard (1974) yields Eq. (14).

$E_{\text {Rear-capture }}=\frac{1}{3 \times 10^{7}} R e_{\text {drop }} \times S t_{\text {ap }}^{-1.23}$

This correlation is presented in solid lines in Fig. 13 and shows a satisfactory agreement with Beard's simulations (crosses) in the corresponding range of the drop Reynolds number and particle Stokes number. However, it should be kept in mind that this relationship is only valid for the drop Reynolds numbers larger than 20 (a $280 \mu \mathrm{m}$ drop at its terminal velocity), since below this critical value there is no recirculating flow behind the drop (Le Clair et al., 1972). Finally, this new contribution should be added to those presented in Table 1 for raindrops.

\section{Conclusions}

This study is a follow up of the paper by Quérel et al. (2014b) and treats questions raised therein. In particular, Quérel et al. (2014b) showed that their efficiency measurements of submicron particles could only be explained by rear capture. The present paper confirms the impact of recirculating flows at the rear of the drop on the collection of submicron particles. This was done by directly comparing our measurements against the numerical simulations of Beard (1974). The BERGAME experimental facility was optimised to considerably reduce the measurement uncertainties, as well as to perfectly control the electric charges of both the drops and the aerosol particles.

As in Quérel et al. (2014b), we show that the collection efficiency of the accumulation mode aerosol particles by drops representative of rain varies significantly with the size of the particles. On a logarithmic scale, the efficiency curve obtained shows a $\mathrm{V}$ shape with a minimum around $0.65 \mu \mathrm{m}$. The increase in the collection efficiency for particles larger than
$0.65 \mu \mathrm{m}$ is attributed to the mechanism of impaction on the front side of the drop. Within this size range, the increase in the diameter of the particle increases its inertia, and the particle can no longer follow the streamlines and thus impacts the drop. It was not possible for the measurements of Quérel et al. (2014b), but here we can directly compare our results with the numerical simulations carried out by Beard (1974). This comparison highlights the robustness of his model for predicting the efficiency of capture of particles by raindrops over the entire accumulation mode. It should be noted that it is the only model to predict the significant increase in the collection efficiency that we measured for submicron particles. This is related to the fact that Beard (1974) first simulated the flow around the drop by solving the complete Navier-Stokes equation (without ignoring the convection terms; Beard and Grover, 1974). Therefore, he captured the separation of the boundary layer at the rear of the drop and the resulting recirculating flows; then, he simulated the trajectory of the particles in this velocity field. Beard thus showed that the increase in the collection efficiency of submicron particles as observed in experiments is due to the fact that these particles are captured in the recirculating flows to the rear of the drop and drawn back into its rear side.

Furthermore, we have also shown that, for particles larger than $1 \mathrm{~mm}$, the models of Beard and Slinn are very similar. Finally, we propose a new semi-analytical expression to calculate the elementary efficiency of capture by the rear recirculating flows. It is important that this mechanism should be systematically taken into account to avoid errors of at least 2 orders of magnitude on the collection efficiency and consequently on the scavenging coefficient.

In the near future, we plan to integrate these new measurements within the DESCAM model (Flossmann, 1986, 1991; Querel et al., 2014a) and to compare the scavenging coefficient derived from the theoretical approaches and the ex- 
periments conducted in the environment by Volken and Shuman (1993), Laakso et al. (2003) and Chate (2005).

Finally, we plan, in a more distant future, to look at other hydrometeors such as snow and hail. 


\section{Appendix A: Evaluation of uncertainties}

The collection efficiency is calculated by means of Eqs. (5) and (8) from which we derive the equation below by substitution:

$E\left(d_{\text {aero }}, D_{\text {drop }}, \mathrm{RH}\right)=\frac{2 D_{\text {Drop }} \cdot[\text { fluo }]_{\text {drop }} \cdot V_{\text {chamber }}}{3 H \cdot[\text { fluo }]_{\text {filter }} \cdot V_{\text {sol }}}$.

The expanded relative measurement uncertainty of the collection efficiency $\left(U_{R, E}\left(d_{\text {aero }}, D_{\text {Drop }}, \mathrm{RH}\right)\right)$ is determined with the help of the law of propagation of variances, considering an expansion factor of 2 (Lira, 2002):

$\mathrm{U}_{R, E}\left(d_{\text {aero }}, D_{\text {Drop }}, \mathrm{RH}\right)$

$=2 \sqrt{u_{R, D_{\text {drop }}}^{2}+\mathrm{u}_{R, \text { ffuoldrop }}^{2}+\mathrm{u}_{R, V_{\text {chamber }}}^{2}+\mathrm{u}_{R, H}^{2}+\mathrm{u}_{R,[\text { fluo }]_{\text {fluter }}}+\mathrm{u}_{R, V_{\text {sol }}}^{2}}$.

In the right-hand member of this expression, the terms $\mathrm{u}_{R, X}$ correspond to the relative measurement uncertainty of $X$. Each experimental uncertainty is discussed in a separate subsection.

\section{A1 Uncertainty in drop size}

Shadowgraph measurements of the size of the drops have shown that our drop generation system is very stable and reproducible for the parameters adopted (Sect. 2.1). The standard deviation of the drop size distribution is $20 \mu \mathrm{m}$; we use this standard deviation to determine the relative uncertainty in the diameter of the drops.

$\mathrm{u}_{R, D_{\text {drop }}}=\frac{\sigma_{D_{\text {drop }}}}{D_{\text {drop }}}=\frac{20 \times 10^{-3}}{1.3} \approx 0.015$

\section{A2 Uncertainty in fluorescein concentration measurements}

For the range of concentrations within which fluorescence spectrometry is used, the calibration certificate of the spectrometer indicates an expanded relative measurement uncertainty $\left(U_{\mathrm{R},[\text { fluo] }}\right)$ of less than $5 \%$.

We then derive the relative measurement uncertainty of the fluorescein concentration in the drops $\left(\mathrm{u}_{R,[f l u o]_{\text {drop }}}\right)$. This relative uncertainty has two contributions. The first one is due to the spectrometer relative measurement uncertainty on the fluorescein concentration $\left(u_{\mathrm{R},[\mathrm{fluo}]}\right)$, and the second one is due to a potential variation of the volume of water collected (in the drop collector; Fig. 7) due to vaporisation during the experiments $\left(u_{\left.R, V_{\text {collected }}\right)}\right)$.

$\mathrm{u}_{R,[\text { fluo }]_{\text {drop }}}=\sqrt{\left(u_{\mathrm{R},[\text { fluo }]}\right)^{2}+\left(u_{R, V_{\text {collected }}}\right)^{2}}$

$u_{\mathrm{R},[\text { fluo }]}=\frac{U_{\mathrm{R},[\text { ffuo }]}}{2}=\frac{0.05}{2}$

The uncertainty on the volume of water collected $\left(u_{R, V_{\text {collected }}}\right)$ is estimated with the maximum variation of the volume of liquid water in the drop collector due to vaporisation $\left(\mathrm{EMT}_{V_{\text {collected }}}\right)$

$u_{R, V_{\text {collected }}}=\frac{\mathrm{EMT}_{V_{\text {collected }}}}{3 V_{\text {collected }}}$

In this equation, the volume of water collected ( $\left.V_{\text {collected }}\right)$ is greater than $1 \mathrm{~cm}^{3}$ (Sect. 2.4). The maximum variation of the volume of liquid water in the drop collector $\left(\mathrm{EMT}_{V_{\text {collected }}}\right)$ is evaluated supposing that during the experiment period (Sect. 2.4) the entire volume of the buffer $\left(V_{\text {buffer }}\right)$ becomes saturated with water vapour. This leads to

$$
\begin{aligned}
\operatorname{EMT}_{V_{\text {collected }}} & =\frac{3 M_{\mathrm{H}_{2} \mathrm{O}} P_{\text {sat }}\left(T_{\text {air }}\right) V_{\text {buffer }}}{R T_{\text {air }} \rho_{\text {liquid-water }}} \\
& =1.2 \times 10^{-2} \mathrm{~cm}^{3} .
\end{aligned}
$$

In this equation, $R$ is the perfect gas constant, $P_{\text {sat }}$ is the saturation vapour pressure, $\rho_{\text {liquid-water }}$ is the density of liquid water, $M_{\mathrm{H}_{2} \mathrm{O}}$ is the molar mass of water and $T_{\text {air }}$ the gas temperature in the buffer. The coefficient of 3 in the numerator comes from the fact that the buffer volume is flushed three times during the measurement period (Sect. 2.4).

$\mathrm{u}_{R,[\text { [luo }]_{\text {drop }}}=\sqrt{\left(\frac{0.05}{2}\right)^{2}+\left(\frac{1.2 \times 10^{-2}}{3 \times 1}\right)^{2}} \approx 0.025$

For the fluorescein concentration measured in the aerosol chamber ([fluo $]_{\text {chamber }}$ ), we have the same uncertainty associated with the fluorescence spectrometry measurement. In addition to this measurement uncertainty, there is a second uncertainty associated with the reduction in concentration during the course of the experiment. We have calculated this reduction to be less than $8 \%$ over the duration of the measurement. The total relative uncertainty in the fluorescein concentration inside the aerosol chamber is therefore approximately $8 \%$ (equation below).

$u_{R,[\text { [fluo }]_{\text {chamber }}}=\sqrt{0.025^{2}+0.08^{2}} \approx 0.08$

\section{A3 Uncertainty in height of aerosol chamber}

The aerosol chamber measures $1.3 \mathrm{~m}$ (plus or minus $1 \mathrm{~mm}$ ). However, over the duration of the measurement, the particles diffuse and move slightly outside the geometric boundaries of the aerosol chamber. We calculate the maximum error in the height of interaction between the drops and the particles $\left(\mathrm{EMT}_{\mathrm{H}}\right.$ ) to be approximately $2 \mathrm{~cm}$ (one above and one below the chamber). We therefore calculate the relative uncertainty for this height of interaction $\left(\mathrm{u}_{R, H}\right)$ by means of the following equation:

$\mathrm{u}_{R, H}=\frac{\mathrm{EMT}_{\mathrm{H}}}{3 H} \approx 0.005$. 


\section{A4 Uncertainty in volume of dilution}

The uncertainty in the volume of dissolution is very low; we estimate its maximum error $\left(\mathrm{EMT}_{V_{\text {sol }}}\right)$ to be $1 \mathrm{~mL}$. We derive a relative uncertainty in the dilution $\left(u_{R, V_{\text {sol }}}\right)$ :

$u_{R, V_{\mathrm{sol}}}=\frac{\mathrm{EMT}_{V_{\mathrm{sol}}}}{3 V_{\mathrm{sol}}} \approx 0.003$.

\section{A5 Uncertainty in volume of aerosol chamber}

The uncertainty in the volume of the aerosol chamber is low, we estimate its maximum error $\left(\mathrm{EMT}_{V_{\text {chambre }}}\right)$ to be $20 \mathrm{cL}$. We derive the relative uncertainty in the dilution $\left(u_{R, V_{\text {chamber }}}\right)$ :

$u_{R, V_{\text {chamber }}}=\frac{\mathrm{EMT}_{V_{\text {chamber }}}}{3 V_{\text {chamber }}}=\frac{20 \times 10^{-2}}{3 \times 10} \approx 0.007$.

\section{A6 Uncertainty in relative humidity}

The relative humidity is not directly involved in the calculation of collection efficiency. However, it is established, for the finest droplets, that the efficiency increases considerably when the relative humidity reduces, due to diffusiophoresis. For example, Grover et al. (1977) calculated that the collection efficiency of a $0.5 \mu \mathrm{m}$ aerosol particle by a $80 \mu \mathrm{m}$, can increase by a factor of $10^{4}$ when the relative humidity falls from 100 to $20 \%$.

However, our recent measurements, for the largest hydrometeors forming rain (between 2 and $2.6 \mathrm{~mm}$; Quérel et al., 2014b) showed no dependency of the collection efficiency on relative humidity.

During our experiments, the aerosol generator settings were optimised in such a way that, at the end of the aerosol chamber filling phase, the relative humidity in the chamber was $75 \pm 1 \%$.

For each measurement, during the 10 min needed to collect $1 \mathrm{~mL}$ of drops (Sect. 2), the relative humidity increased by $5 \pm 1 \%$. This increase is related to an accumulation of water on the slightly inclined bottom of the aerosol chamber.

We consider therefore that the measurement uncertainty for the relative humidity is approximately $5 \%$. 
Competing interests. The authors declare that they have no conflict of interest.

Acknowledgements. We give special thanks to Denis Boulaud for his confidence and support.

Edited by: B. Ervens

Reviewed by: two anonymous referees

\section{References}

Ardon-Dryer, K., Huang, Y.-W., and Cziczo, D. J.: Laboratory studies of collection efficiency of sub-micrometer aerosol particles by cloud droplets on a single-droplet basis, Atmos. Chem. Phys., 15, 9159-9171, doi:10.5194/acp-15-9159-2015, 2015.

Andronache, C., Gr"nholm, T., Laakso, L., Phillips, V., and Venäläinen, A.: Scavenging of ultrafine particles by rainfall at a boreal site: observations and model estimations, Atmos. Chem. Phys., 6, 4739-4754, doi:10.5194/acp-6-4739-2006, 2006.

Baron, P. A.: Calibration and use of the aerodynamic particle sizer (APS 3300), Aerosol Sci. Technol., 5, 55-67, 1986.

Beard, K. V.: Experimental and numerical collision efficiencies for submicron particles scavenged by small raindrops, J. Atmos. Sci., 31, 1595-1603, 1974.

Beard, K. V. and Grover, S. N.: Numerical collision efficiencies for small raindrops colliding with micron size particles, J. Atmos. Sci., 31, 543-550, 1974.

Beard, K. V.: Terminal velocity and shape of cloud and precipitation drops aloft, J. Atmos. Sci., 33, 851-864, 1976.

Beard, K. V. and Chuang, C.: A new model for the equilibrium shape of raindrops, J. Atmos. Sci., 44, 1509-1524, 1987.

Charlson, R. J., Schwartz, S. E., Hales, J. M., Cess, R. D., Hansen, J. E., and Hofmann, D. J.: Climate Forcing by Anthropogenic Aerosols, Science, 255, 423-430, 1992.

Chate, D. M.: Study of scavenging of submicron-sized aerosol particles by thunderstorm rain events, Atmos. Environ., 39, 66086619, 2005.

Davenport, H. M. and Peters, L. K.: Field studies of atmospheric particulate concentration changes during precipitation, Atmos. Environ., 12, 997-1008, 1978.

Depuydt, G.: Etude expérimentale in situ du potentiel de lessivage de l'aérosol atmosphérique par les précipitations, Doctoral dissertation, available from Toulouse University, 2013.

Depuydt, G., Masson, O., Gomes, L., and Brenguier, J. L.: Micro and macro-physical characterizations of precipitations in continental and Mediterranean environments, in: 16th International Conference on Clouds and Precipitation, ICCP-2012, July 30August 3 2012, Leipzig, Germany, 2012.

Flossmann, A. I.: A theoretical investigation of the removal of atmospheric trace constituents by means of a dynamic model, $\mathrm{PhD}$ thesis, Phys. Dep., Johannes Gutenberg-Univ. Mainz, Mainz, Germany, 186 pp., 1986.

Flossmann, A. I.: The scavenging of two different types of marine aerosol particles calculated using a two-dimensional detailed cloud model, Tellus, 43B, 301-321, 1991.

Fritsch, F. N. and Carlson, R. E.: Monotone piecewise cubic interpolation, SIAM J. Numer. Anal., 17, 238-246, 1980.
Greenfield, S. M.: Rain scavenging of radioactive particulate matter from the atmosphere, J. Meteorol., 14, 115-125, 1957.

Groëll, J., Quélo, D., and Mathieu, A.: Sensitivity analysis of the modelled deposition of $137 \mathrm{Cs}$ on the Japanese land following the Fukushima accident, Int. J. Environ. Pollut., 55, 67-75, 2014.

Grover, S. N., Pruppacher, H. R., and Hamielec, A. E.: A numerical determination of the efficiency with which spherical aerosol particles collide with spherical water drops due to inertial impaction and phoretic and electrical forces, J. Atmos. Sci., 34, 1655-1663, 1977.

Hampl, V. M. D. D. E., Kerker, M., Cooke, D. D., and Matijevic, E.: Scavenging of aerosol particles by a falling water droplet, J. Atmos. Sci., 28, 1211-1221, 1971.

Hinds, W. C., Ashley, A., Kennedy, N. J., and Bucknam, P.: Conditions for cloud settling and Rayleigh-Taylor instability, Aerosol Sci. Technol., 36, 1128-1138, 2002.

Jaenicke, R.: Aerosol physics and chemistry, Zahlenwerte und Funktionen aus Naturwissenschaften und Technik, 4, 391-457, 1988.

Kerker, M. and Hampl, V.: Scavenging of Aerosol Particles by a Failing Water Drop and Calculation of Washout Coefficients, J. Atmos. Sci., 31, 1368-1376, 1974.

Laakso, L., Grönholm, T., Rannik, Ü., Kosmale, M., Fiedler, V., Vehkamäki, H., and Kulmala, M.: Ultrafine particle scavenging coefficients calculated from 6 years field measurements, Atmos. Environ., 37, 3605-3613, 2003.

Ladino Moreno, L. A., Stetzer, O., and Lohmann, U.: Contact freezing: a review of experimental studies, Atmos. Chem. Phys., 13, 9745-9769, doi:10.5194/acp-13-9745-2013, 2013.

Lai, K. Y., Dayan, N., and Kerker, M.: Scavenging of aerosol particles by a falling water drop, J. Atmos. Sci., 35, 674-682, 1978.

Le Clair, B. P., Hamielec, A. E., Pruppacher, H. R., and Hall, W. D.: A theoretical and experimental study of the internal circulation in water drops falling at terminal velocity in air, J. Atmos. Sci., 29, 728-740, 1972.

Lira, I.: Evaluating the Measurement Uncertainty: Fundamentals and Practical Guidance, Taylor \& Francis, Institute of Physics, Bristol, UK, 2002.

Marjamäki, M., Keskinen, J., Chen, D. R., and Pui, D. Y.: Performance evaluation of the electrical low-pressure impactor (ELPI), J. Aerosol Sci., 31, 249-261, 2000.

Ménard, T., Tanguy, S., and Berlemont, A.: Coupling level set/VOF/ghost fluid methods: Validation and application to 3D simulation of the primary break-up of a liquid jet, Int. J. Multiphas. Flow, 33, 510-524, 2007.

Mircea, M. and Stefan, S.: A theoretical study of the microphysical parameterization of the scavenging coefficient as a function of precipitation type and rate, Atmos. Environ., 32, 2931-2938, 1998.

Mircea, M., Stefan, S., and Fuzzi, S.: Precipitation scavenging coefficient: influence of measured aerosol and raindrop size distributions, Atmos. Environ., 34, 5169-5174, 2000.

Park, S. H., Jung, C. H., Jung, K. R., Lee, B. K., and Lee, K. W.: Wet scrubbing of polydisperse aerosols by freely falling droplets, J. Aerosol Sci., 36, 1444-1458, 2005.

Pranesha, T. S. and Kamra, A. K.: Scavenging of aerosol particles by large water drops: 1 . Neutral case, J. Geophys. Res.-Atmos., 101, 23373-23380, 1996. 
Pruppacher, H. R. and Beard, K. V.: A wind tunnel investigation of the internal circulation and shape of water drops falling at terminal velocity in air, Q. J. Roy. Meteorol. Soc., 96, 247-256, 1970.

Pruppacher, H. R., Klett, J. D., and Wang, P. K.: Microphysics of clouds and precipitation, Kluwer academic publishers, Dordrecht, Boston, London, 852 pp., 1998.

Quérel, A., Monier, M., Flossmann, A. I., Lemaitre, P., and Porcheron, E.: The importance of new collection efficiency values including the effect of rear capture for the below-cloud scavenging of aerosol particles, Atmos. Res., 142, 57-66, 2014a.

Quérel, A., Lemaitre, P., Monier, M., Porcheron, E., Flossmann, A. I., and Hervo, M.: An experiment to measure raindrop collection efficiencies: influence of rear capture, Atmos. Meas. Tech., 7, 1321-1330, doi:10.5194/amt-7-1321-2014, 2014b.

Quérel, A., Roustan, Y., Quélo, D., and Benoit, J. P.: Hints to discriminate the choice of wet deposition models applied to an accidental radioactive release, Int. J. Environ. Pollut., 58, 268-279, 2015.

Slinn, W. G. N.: Some approximations for the wet and dry removal of particles and gases from the atmosphere, Water Air Soil Pollut., 7, 513-543, 1977.

Sow, M. and Lemaitre, P.: Influence of electric charges on the washout efficiency of atmospheric aerosols by raindrops, Ann. Nucl. Energy, 93, 107-113, 2016.

Stöber, W. and Flachsbart, H.: An evaluation of nebulized ammonium fluorescein as a laboratory aerosol, Atmos. Environ., 7, 737-748, 1973.

Szakáll, M., Diehl, K., Mitra, S. K., and Borrmann, S.: A wind tunnel study on the shape, oscillation, and internal circulation of large raindrops with sizes between 2.5 and $7.5 \mathrm{~mm}$, J. Atmos. Sci., 66, 755-765, 2009.

Szakáll, M., Mitra, S. K., Diehl, K., and Borrmann, S.: Shapes and oscillations of falling raindrops - A review, Atmos. Res., 97, 416-425, 2010.

Tinsley, B. A., Rohrbaugh, R. P., Hei, M., and Beard, K. V.: Effects of image charges on the scavenging of aerosol particles by cloud droplets and on droplet charging and possible ice nucleation processes, J. Atmos. Sci., 57, 2118-2134, 2000.
Tinsley, B. A., Zhou, L., and Plemmons, A.: Changes in scavenging of particles by droplets due to weak electrification in clouds, Atmos. Res., 79, 266-295, 2006.

Tinsley, B. A. and Zhou, L.: Parameterization of aerosol scavenging due to atmospheric ionization, J. Geophys. Res.-Atmos., 120, 8389-8410, 2015.

Twomey, S.: Pollution and the planetary albedo, Atmos. Environ., 8, 1251-1256, 1974.

Vohl, O., Mitra, S. K., Wurzler, S. C., and Pruppacher, H. R.: A wind tunnel study of the effects of turbulence on the growth of cloud drops by collision and coalescence, J. Atmos. Sci., 56, 40884099, 1999.

Volken, M. and Schumann, T.: A critical review of below-cloud aerosol scavenging results on Mt. Rigi, Water Air Soil Pollut., 68, 15-28, 1993.

Waldmann, L.: Über die Kraft eines inhomogenen Gases auf kleine suspendierte Kugeln, Z. Naturforsch., 14, 589-599, 1959.

Wang, P. K. and Pruppacher, H. R.: An experimental determination of the efficiency with which aerosol particles are collected by water drops in subsaturated air, J. Atmos. Sci., 34, 1664-1669, 1977a.

Wang, P. K. and Pruppacher, H. R.: Acceleration to terminal velocity of cloud and raindrops, J. Appl. Meteorol., 16, 275-280, $1977 b$.

Wang, R., Tao, S., Shen, H., Huang, Y., Chen, H., Balkanski, Y., Boucher, O., Ciais, P., Shen, G. F., Li, W., Zhang, Y. Y., Chen, Y. C., Lin, N., Su, S., Li, B. G., Liu, J. F., and Liu, W. X.: Trend in global black carbon emissions from 1960 to 2007, Environ. Sci. Technol., 48, 6780-6787, 2014.

Wang, X., Zhang, L., and Moran, M. D.: Uncertainty assessment of current size-resolved parameterizations for below-cloud particle scavenging by rain, Atmos. Chem. Phys., 10, 5685-5705, doi:10.5194/acp-10-5685-2010, 2010.

Whitby, K. T.: On the multimodal nature of atmospheric aerosol size distribution, in: VIII International Conference on Nucleation, Leningrad, USSR, September, 24-29, 1973. 